\title{
Observations on the reflex effects seen in Parkinson's disease on terminating a period of tendon vibration
}

\author{
P B C Matthews, F W J Cody, Helen C Richardson, N MacDermott
}

\begin{abstract}
Vibration was applied to the tendon of flexor carpi radialis while recording the EMG of the wrist flexors in 29 Parkinsonian patients. Cessation of the vibration led to a small short-latency (approximately $25 \mathrm{~ms}$ ) reduction in the level of activity which did not differ in magnitude from the normal. Moreover, there was no sign of any subsequent long-latency reduction of activity. Thus the maintained tonic activity of Parkinsonian muscles seems unlikely to be due to an enhancement of the tonic reflex actions of the Ia afferents, especially via the shortlatency pathway. In addition, the findings argue against reduction of either $\mathrm{Ia}$ or $\mathbf{I b}$ firing being responsible for the delayed excitatory "Westphal" (or "shortening") response that may occur in parkinsonism on allowing a muscle to shorten; this was never found on terminating vibration, even when present on muscle release.
\end{abstract}

The reflex effects produced on the wrist flexors by the onset of a period of tendon vibration were recently analysed electromyographically in a number of patients with Parkinson's disease to investigate the role of autogenetic reflexes in the production of rigidity. ${ }^{1}$ The sole effect was a short-latency excitation which did not differ from the normal. This contrasted with an augmentation of the long-latency component of the response elicited by stretching the muscle at moderate velocity, ${ }^{12}$ but is in line with repeated studies on the tendon jerk which has long been known to be unaltered in Parkinson's disease in spite of the increase in muscle tone (most recently by Rothwell et $a l^{2}$ ). Vibration, like tendon taps, strongly excites the Ia afferents from the muscle spindle primary endings and provides an alternative way of testing the state of the reflex pathways activated by a Ia input. In both cases, however, other afferents will also be excited to some degree; this slightly complicates the issue, but increases the value of such complementary studies since such unwanted actions are likely to differ in the two cases.

Vibration has an advantage over taps in that it allows the strength of the tonic spinal action of short-latency pathways, activated by a maintained Ia input, to be assessed by determining the immediate reduction of the level of EMG activity after vibrating; the size of any maintained tonic vibration reflex potentially depends also upon multiple long-latency res- ponses, including "voluntary" action. Reducing Ia firing by stopping vibration is rather different from achieving the same effect by moving a limb to shorten the muscle studied, since muscle spindle secondary endings should no longer be significantly affected nor the antagonists stretched. Tonic mechanisms merit study per se, since it is common knowledge that the tone of a muscle and the briskness of its jerk can vary independently; several neurophysiological mechanisms are known which could cause this. The previous work has thus been extended by examining the reflex effect on a muscle ceasing to vibrate its tendon, after having maintained the vibration long enough for the phasic responses on its initiation to have died away. As before, ${ }^{12}$ the state of the reflex pathways was examined while the subject was maintaining a weak steady voluntary contraction rather than simply exhibiting resting rigidity; however, a derangement of reflex action contributing significantly to the genesis of rigidity would still be expected to manifest itself.

We were also interested to see whether a delayed excitatory "Westphal" response would occur on ceasing to vibrate, as would be expected if it were to be a rebound effect triggered by reducing the Ia firing. This "paradoxical" response in humans was first described by Westphal in $1880,{ }^{3}$ yet remains poorly understood. It is the inverse of the stretch reflex and typically consists of an abrupt reflex excitation of a muscle on displacing the limb to allow the muscle to shorten and it occurs commonly in patients with Parkinson's disease. ${ }^{4-8}$ It is often referred to as the "shortening reaction" by supposed homology with the effect given this name by Sherrington' in 1909, which he observed in decerebrate and chronic spinal preparations and which Rademaker ${ }^{10}$ subsequently saw in humans. However, the effects typically described for Parkinsonism differ sufficiently from Sherrington's shortening reaction to raise serious doubts as to whether they are in fact the same thing. Thus the term "Westphal reaction" will be retained here. ${ }^{411}$ The Westphal response was also sought directly in our subjects by examining the effects of allowing the muscle to shorten when contracting.

\section{Methods}

Observations were made on 29 patients having treatment for Parkinson's disease and 12 normal subjects, each of whom gave informed consent. The average age of the patients was
Received 21 February 1989 and in revised form

Accepted 19 September 1989 
slightly greater than that of the normal subjects (62 and 54 years respectively); the time since diagnosis of the patients' parkinsonism varied from six months to 22 years (mean $7 \cdot 4$ years). The patients all showed clinical rigidity, while remaining on their standard medication (normally levodopa with carbidopa, "Sinemet"). As before, ${ }^{1}$ the rigidity at the wrist was assessed clinically at the time of the experiment and classified on a standard four point scale $e^{12}(0$, absent, normal tonus; 1 , slight but definite-16 patients; 2 , moderate- 10 patients; 3 , severe3 patients). All the patients selected had sufficient voluntary control to perform the experiment, which entailed maintaining a target force by wrist flexion for two minutes on a number of occasions; this also required the absence of appreciable tremor. The patients also had sufficient voluntary control to be able to continue to live at home, rather than being permanently institutionalised. Thus, this study has been carried out on patients with mild to moderate symptoms, rather than those with severe disabilities.

The methods used have been described previously. ${ }^{1}$ The only significant difference was in the duration of the periods of stretch and of vibration employed. In the previous experiments successive responses to the onset of vibration were averaged for analysis, so brief periods of vibration were delivered followed by a longer recovery period. In the present experiments, on the effect of stopping vibration, the timing was reversed and a maintained period of vibration was briefly interrupted, with the "recovery" taking place during the continuation of the stimulus.

To save referral to the previous paper for the rest of the methodology, the essentials of the methods described previously are given below. The subjects grasped a handle by means of which "releases" could be applied, and which incorporated a force transducer. They were instructed to flex the wrist with a constant voluntary effort to produce about $20 \%$ of the maximal voluntary contractile force, and to ignore the stimuli. The same target force was used in the presence and absence of maintained vibration. However, the quasi-steady level of EMG activity was $20-30 \%$ higher in the former case; this probably arose largely from increased synchronisation of motor unit discharge, induced by the vibration, leading to more effective summation of their potentials. ${ }^{13}$ The release was about $10^{\circ}$ wrist displacement, normally at $50-200^{\circ} \mathrm{s}-1$. Vibration at $123 \mathrm{~Hz}$ of 0.7 or $1.0 \mathrm{~mm}$ peak-to-peak amplitude was applied percutaneously to the tendon of flexor carpi radialis $5 \mathrm{~cm}$ above the wrist crease. The electromechanical vibrator incorporated a position transducer so that its movement could be monitored, as was that of the separate servocontrolled stretcher. The electrical activity of flexor carpi radialis was recorded with surface electrodes placed $5 \mathrm{~cm}$ apart over its belly; the resulting EMG will also have reflected the activity of other wrist flexors. The EMG was rectified, slightly smoothed (1 ms time constant) and then averaged (bin-width $0.4 \mathrm{~ms}$ ) to produce records for study. Normally each particular stimulus pattern was studied once only.

\section{Details of timing}

These are described at length, since they may influence the results obtained. ${ }^{14}$ The interval between successive terminations of a given stimulus was varied in a pseudo-random manner between 1.0 and $1.5 \mathrm{~s}$ (mean rate close to $0.8 \mathrm{~Hz}$ ). The cycle was repeated for just over 2.5 minutes to provide 128 samples for averaging; the gap in the otherwise continuous vibration lasted for $200 \mathrm{~ms}$, as did the release of the stretch (this includes both the period during which the muscle was shortening, which varied with the velocity employed, and also the time it was held at its shortened length; it does not include the subsequent period of re-extension). This cycle was probably too rapid to have allowed the reflex centres to have settled to a completely steady state during the maintenance of the stretch or vibration. A relatively high repetition rate was required to obtain sufficient samples to allow the "off" responses to stand out in the averages, but such rates do not prevent the production of large delayed unloading responses by the digital flexors. ${ }^{15} 16$ Control experiments on five normal subjects showed little change in the present "off" responses on lowering the mean cycle frequency successively to $0.4,0.13$ and $0.08 \mathrm{~Hz}$ (rounded values), and there was no sign of a progressive development of a delayed long-latency component.

When the responses to the onset of vibration were recorded the cycle reverted to that used before, ${ }^{\prime}$ with brief periods of vibration separated by a recovery period; the mean cycle rate was then $0.4 \mathrm{~Hz}$ and 64 trials were averaged. On a number of occasions (fig 1,3 ), the averages obtained with our normal rates of stimulation were tested for statistical stationarity by comparing them with those obtained from the first and second halves of the same 2.5 minutes period of data collection; no differences were then apparent showing that the responses studied were stable and resistant to "habituation". 14

\section{Results}

Figure 1 illustrates the typical effect of stopping vibration, both for a normal subject and for a patient with Parkinson's disease who had appreciable rigidity at the wrist on clinical testing. The responses to the onset of vibration are shown below. As before, there is no significant difference between the "on" responses; neither shows any sign of a long-latency excitation, corresponding to that which occurs with stretch at about $50 \mathrm{~ms}$, immediately following the short-latency discharge. ${ }^{1}$ Likewise, there is no appreciable difference between the "off" responses in the two cases; both show a reduction of EMG activity beginning 25-30 ms after the last peak of vibration. This is clearly a "short-latency" effect since the latency of the "on" response was about $25 \mathrm{~ms}$. The "off" effect can reasonably be attributed to a disfacilitation arising from the removal of an 
a Normal

(b) Parkinson's disease
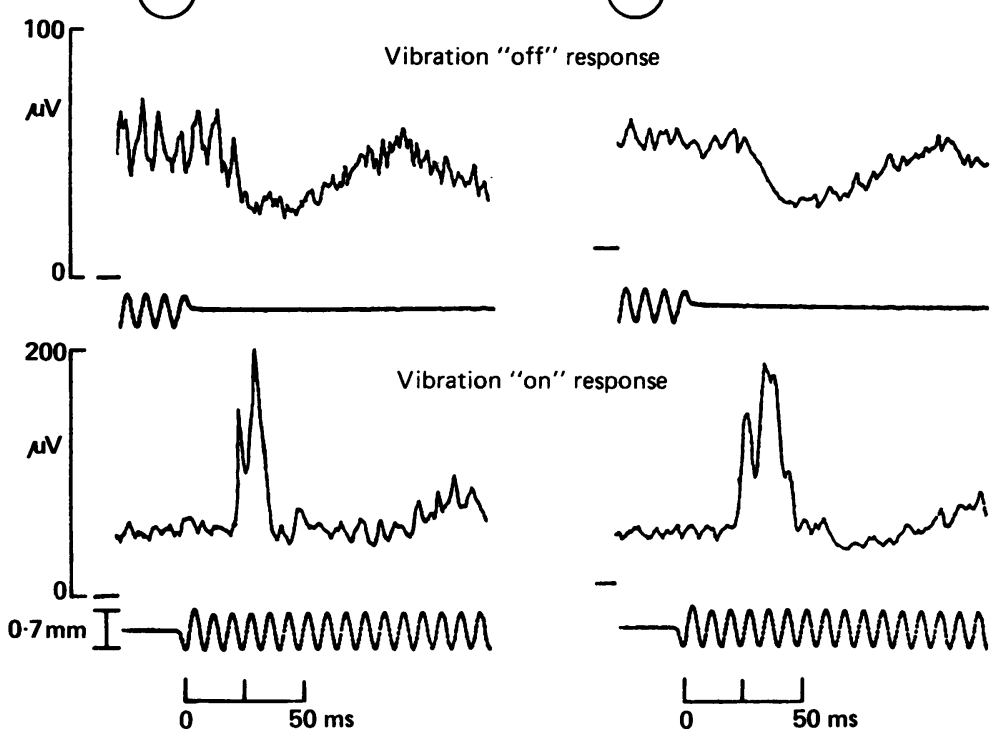

Figure 1 Comparison of the responses occurring at the beginning and at the end of vibration. Left, for a normal subject; right for a patient with Parkinson's disease with grade 1 rigidity. The surface EMG was rectified and then averaged. The EMG traces have been mounted so that their initial levels are aligned; their zeros are given by the short horizontal bars; the upper records are on twice the scale of the lower records. For all records the initial level of activity corresponded to approximately $20 \%$ of maximal voluntary contraction; the responses can thus be seen to be of appreciable physiological magnitude. The lower traces show the movement of the vibrator. The "on" and "off" responses were recorded in response to separate periods vibrator. The "on" and "off" responses were recorded in response to separate
of vibration (see methods) and different numbers averaged ( $N=64$ and 128 respectively). sizes of the "on" and "off" responses are highly correlated (Pearson's correlation coefficient $=0.53, \mathrm{p}<0.001)$. The normal and the Parkinsonian responses overlap almost completely and no systematic difference was found between the two populations on statistical testing (see below). As already noted, ${ }^{1}$ the similarity of the responses at the onset of vibration in the Parkinsonian patients and in the normal group agrees with the view that vibration tests the state of the same shortlatency reflex pathways as does the tendon jerk. The new finding is that there is no difference in the effect of removing a maintained excitatory input, thereby demonstrating that the tonic as well as the phasic behaviour of the shortlatency pathway remains unaltered in parkinsonism. Nor was there ever any indication of a tonic Ia input producing a tonic long-latency excitation.

\section{Statistics}

No significant difference (at the 5\% level) was found between the Parkinsonian patients and the normal group on using the $t$ test to compare the means of either the "on" or the "off" responses; nor was one found on employing the non-parametric Mann-Whitney U test to compare the two populations. These statistical comparisons were also performed for the responses elicited by each amplitude of vibration on its own $(0.7$ and $1.0 \mathrm{~mm})$. Again there was no difference between the two populations, except for a questionable small reduction $(20 \%)$ in the size of the "off" effect with 0.7 $\mathrm{mm}$ vibration for the parkinsonian patients; this was just significant (at the $5 \%$ level) on the

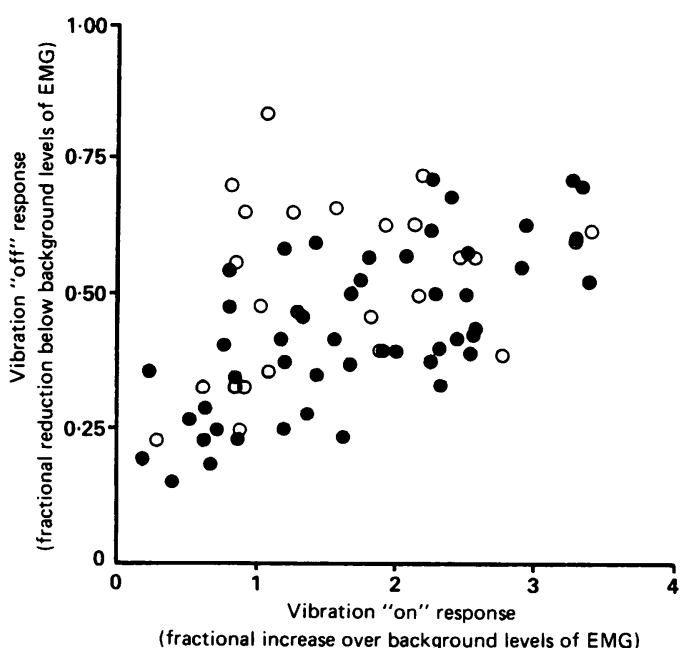

Figure 2 Scatter diagram comparing the effects at the beginning and end of vibration for 29 parkinsonian patients (O) and 12 normal subjects ( $O$ ). The individual responses for both "on" and "off" were determined by taking the difference between the maximum effect (measured over 1-3 bins at the top of the peak and the bottom of the trough, respectively) and the preexisting EMG level (averaged over the $20 \mathrm{~ms}$ immediately following the start or cessation of vibration, respectively, before any reflex change could have occurred). The values were then normalised by expressing them as a proportion of the pre-existing level, as measured above. All the normal subjects and most of the patients have contributed two points to the diagram, one for each of two amplitudes of vibration $(0.7$ and $1.0 \mathrm{~mm}$ pk-pk, which evoked remarkably similar sized responses at both "on'" and "off"). 
$t$ test, but was not significant on the nonparametric test. These tests were performed on the normalised values of the responses, as in fig 2 . When they were repeated using absolute values of EMG, measured in $\mu \mathrm{V}$, no significant difference was found between the Parkinsonian patients and the normals for the "on" responses, the "off" responses, and the quasi-steady levels during the maintained contractions with and without vibration (measured as for fig 2). In both groups the EMG level during vibration was slightly higher during maintained vibration than in its absence (see Methods); when the "off" responses were normalised in relation to the EMG level in the absence of vibration there was again no difference between those of the patients and of the normal group. Thus the conclusions are not dependent upon the particular method of normalisation used for fig 2 .

\section{Releases and the Westphal reaction}

Displacement of the wrist in the direction required to release the contracting wrist flexors was normally followed by an obvious reduction of their ongoing EMG activity. Such "unloading" responses are well known and are due to a disfacilitation produced by removing an ongoing excitatory afferent input. They may comprise apparently distinct components of different latency, the relative preponderance of which may vary from muscle to muscle. ${ }^{16}$ The short-latency component was dominant for most of our subjects, whether normal or diseased; however, in both groups a longlatency component was sometimes identifiable corresponding to that already described for stretch, and it occasionally occurred in the absence of a preceding short-latency unloading response. As a population, the Parkinsonian patients did not manifest any grossly obvious enhancement of the long-latency component, but the matter was not examined in detail.

The present responses to release were suspected to represent the net effect of several interacting reflexes. Among these may be the Westphal reaction, which consists of excitation of a muscle on displacing a joint in the direction

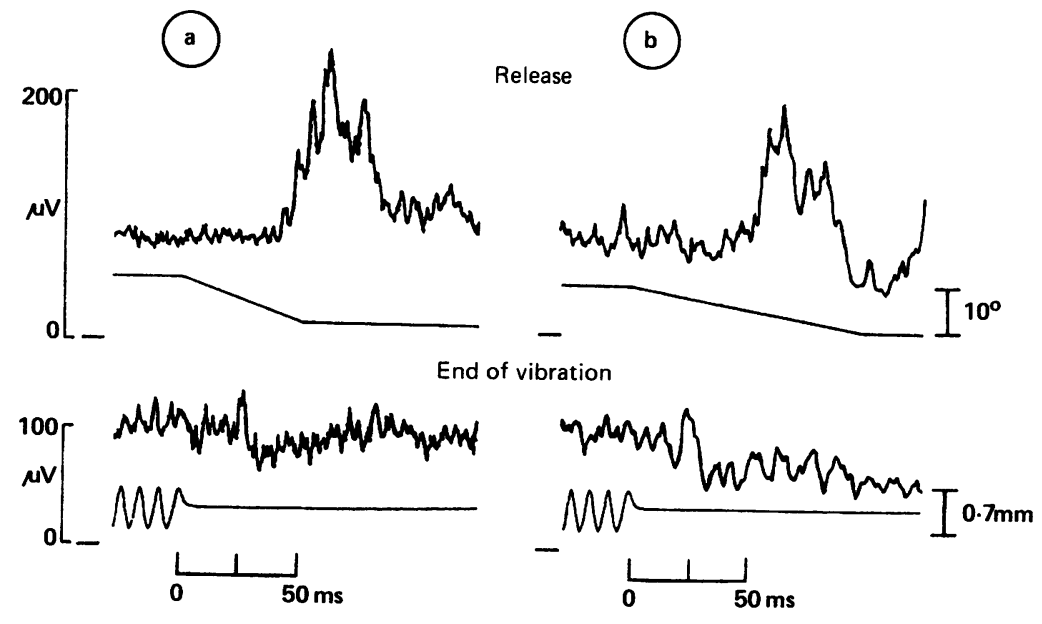

Figure 3 Top, the paradoxical excitatory Westphal response seen in two Parkinsonian patients on unloading the contracting muscle. Bottom, the absence of a corresponding excitation on terminating vibration. (Details as in figure 1). that allows the muscle studied to shorten (see Introduction). Any such Westphal excitation must counteract the disfacilitation normally produced by releasing a contracting muscle and vice versa. Well developed Westphal responses were rare in our series, perhaps because of such interactions. Their relatively paucity may perhaps have been related to the relatively high stimulus repetition rate $(0.8 \mathrm{~Hz})$ or to the muscle being released from a state of steady contraction under voluntary drive, rather than from being "at rest". However, definite Westphal responses were observed in five of the 29 Parkinsonian patients and one of the 12 normal subjects. Figure 3 (top) shows clear-cut examples from two separate patients; like the other definite cases, the latency corresponds to that of a long, rather than of a short latency stretch response. Any unloading response would seem to have been overwhelmed by the Westphal response, as already noted by Rondot and Metral. ${ }^{8}$ For other patients, the superposition of a smaller Westphal response upon the standard unloading response could well have been sufficient to prevent its recognition.

Figure 3 primarily illustrates a quite new finding, namely that a "Westphal" response is not evoked by the cessation of a period of vibration, even when the central state of the patient favours its occurrence. Neither of the bottom records shows any sign of a response corresponding to the large Westphal responses of the upper records from the same patients; the small transitory elevations at $20-25 \mathrm{~ms}$ are close to the noise level and may well depend upon adventitious factors (such as a changing synchrony of the unitary EMG responses). Likewise, none of the other three Parkinsonian patients with a Westphal response on release showed a corresponding excitation on the termination of vibration. Nor was any hint of one found for any of the remaining 29 patients or for the 12 normal subjects. The rate of removal of Ia activity on cessation of vibration is likely to have been appreciably greater than that on release of stretch. This, however, seems unlikely to be responsible for the absence of a Westphal reaction with vibration, since its occurrence on release of stretch tended to be favoured by increasing the rate of release over the range studied $\left(50-200 \operatorname{deg~s}{ }^{1}\right)$.

\section{Discussion}

Tonic response of reflex centres to Ia excitation. Our experiments show that short-latency Ia action once again remained within normal limits in Parkinsonian patients, confirming that derangements in its central pathway cannot be held responsible for the resting tone and rigidity of Parkinsonism. ${ }^{217}$ The new feature is that there has now been found to be no systematic change in the tonic behaviour of the short-latency pathway, as well as no change in its phasic behaviour at the start of stimulation. This was achieved by using vibration; the level of continued tonic activity of the reflex centres in response to a continued Ia input could then be determined simply by ceasing to vibrate, 
and observing the resulting immediate reduction in the level of muscle activity.

In addition, cessation of a period of vibration failed to produce a long-latency reduction of muscle activity. This is entirely in line with the failure of its commencement to produce longlatency excitation ${ }^{1}$, even though stretching a muscle by limb displacement does so. It argues against the tonic activation of long-latency pathways by a Ia input being responsible for the maintained tone of Parkinsonism. However, the matter must remain sub judice pending a better understanding of the contrasting effects of stretch and of vibration in evoking long-latency responses, when both produce Ia excitation.

\section{Westphal reaction.}

Equally of interest was that the elusive Westphal (or shortening) reaction was never seen on terminating vibration, even when it was present on releasing the stretched wrist flexors. This shows that it cannot be due to a reduction of Ia activity by some "rebound" action. Moreover, it also seems unlikely to represent a disinhibition resulting from a reduction of the Ib discharge from the Golgi tendon organs of the muscle being released, as favoured by Berardelli and Hallett. ${ }^{6}$ If it was due to such an action, then it should also have been seen with vibration. During muscle contraction, as in our experiments, tendon organs can be expected to be excited to some degree by vibration, ${ }^{18}$ and should reduce their discharge on its cessation, as they do on the termination of a stretch.

This would seem to leave the discharge of receptors in the stretched antagonist as an obvious source of the Westphal reaction, but these are unlikely to be invariably responsible. In patients with athetosis, Rondot and Scherrer ${ }^{19}$ noted that the Westphal reaction persisted unchanged after infiltrating the antagonist with sufficient local anaesthetic to greatly reduce its motor power. In contrast, for patients with Parkinson's disease, DennyBrown ${ }^{11}$ mentioned that such infiltration might abolish the reaction along with rigidity while movement remained free. Neither account is sufficiently detailed to permit proper assessment; however, it is possible that the Westphal reaction is not a single entity, but due to different mechanisms under different conditions. ${ }^{5}$ Thus it would be premature to generalise on the findings on vibration in Parkinson's disease to cover athetosis. Moreover, cutaneous and/or joint afferents may well sometimes be implicated in the genesis of the Westphal reaction. Their excitation on joint rotation elicits a response of appropriate latency from the deep finger flexor of normal subjects, ${ }^{16}$ and cutaneous reflexes are enhanced in many patients with good Westphal responses. ${ }^{8}$ The subject merits further study, since resolution of the origin of the Westphal response seems likely to throw light on the normal operation of the underlying neural circuitry which current animal experimentation is showing to contain a wealth of unexpected connections.

We thank Drs R G Lascelles and D Neary for allowing us to study patients under their care, and the patients themselves for their cheerful cooperation.

1 Cody FWJ, MacDermott N, Matthews PBC, Richardson HC. Observations on the genesis of the stretch reflex in Parkinson's disease. Brain 1986;109:229-49.

2 Rothwell JC, Obeso JA, Traub MM, Marsden CD. The behaviour of the long-latency stretch reflex in patients with Parkinson's disease. J Neurol Neurosurg Psychiatry with Parkinson's

3 Westphal C. Ueber eine Art paradoxer Muskelcontraction Archiv fur Psychiatrie und Nervenkrankheiten 1880 10:243-8

4 Andrews CJ, Burke D, Lance JW. The response to muscle stretch and shortening in Parkinsonian rigidity. Brain 1972;95:795-812.

5 Angel RW. Muscular contractions elicited by passive shortening. Advances in Neurology 1983;39:555-63. 6 Berardelli A, Hallett $M$. Shortening reaction of human

7 Lee RG, Murphy JT, Tatton WG. Long-latency myotatic reflexes in man: mechanisms, functional significance, and changes in patients with Parkinson's disease. Advances in Neurology 1983;39:489-508.

8 Rondot P, Metral S. Analysis of the shortening reaction in man. In: Desmedt JE, ed. New Developments in Electromyography and Clinical Neurophysiology. Basel: Karger, 1973;3:629-34.

9 Sherrington CS. On plastic tonus and proprioceptive reflexes. $Q J$ Exp Physiol 1909;2:109-56.

10 Rademaker GGJ. On the lengthening and shortening reactions and their occurrence in man. Brain 1947;70:109-26.

11 Denny-Brown D. The Basal Ganglia and their Relation to Disorders of Movement. Oxford: Oxford University Press, 1962:144.

12 Lakke JPWF. Classification of extrapyramidal disorders. $J$ Neurol Sci 1981;51:311-27.

13 Matthews PBC. The reflex excitation of the soleus muscle of the decerebrate cat caused by vibration applied to its tendon. J Physiol 1966;184:450-72.

14 Rothwell JC, Day BL, Berardelli A, Marsden CD. Habituation and conditioning of the human long latency stretch reflex. Exp Brain Res 1986;63:197-204

15 Matthews PBC. Evidence from the use of vibration that the human long-latency stretch reflex depends upon spindle secondary afferents. J Physiol 1984;204:365-93.

16 Matthews PBC, Miles TS. On the long-latency reflex responses of the human flexor digitorum profundus. J Physiol 1988;404:515-34.

7 Berardelli A, Sabra AF, Hallett M. Physiological mechanisms of rigidity in Parkinson's disease. J Neurol Neurosurg Psychiatry 1983;46:45-53.

18 Roll JP, Vedel JP, Ribot E. Alteration of proprioceptive messages induced by tendon vibration in man: a micronmessages induced by tendon vibration in man: a micr

19 Rondot P, Scherrer J. Contraction réflexe provoquée par le raccourissement passif du muscle dans l'athétose et les dystonies d'attitude. Rev Neurol Paris 1966;114:329-37. 